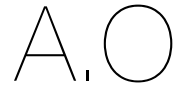

ARTIGO ORIGINAL

1 Escola Superior de

Saúde da Universidade do Algarve,

Campus de Gambelas,

Edifício 1

8005-139 Faro, Portugal

2 Centro de Estudos e Desenvolvimento em

Saúde da Universidade do

Algarve,

Campus de Gambelas,

Edifício 1

8005-139 Faro, Portugal

"Endereço para correspondência:

Maria Palma Mateus Escola Superior de Saúde da

Universidade do Algarve,

Campus de Gambelas,

Edifício 1,

8005-139 Faro, Portugal

mpmateus@ualg.pt

Histórico do artigo:

Recobico a 5 de jutho de 2019 Aceite a 27 de março de 2020

\title{
HÁBITOS ALIMENTARES, DE SAÚDE E ADESÃO À DIETA MEDITERRÂNICA DOS JOVENS DA REGIÃO DO ALGARVE
}

\author{
EATING HABITS, HEALTH AND ADHERENCE TO \\ MEDITERRANEAN DIET AMONG YOUNG PEOPLE IN \\ ALGARVE
}

Joana Margarida Bôto'; Ezequiel Pinto 1,2; Maria Palma Mateus'

RESUMO

INTRODUÇÃo: Estudo desenvolvido pela Universidade do Algarve, inserido no âmbito do Projeto 0290_MEDITA_5_P - "Dieta Mediterrânica Promove Saúde", financiado pelo Fundo Europeu de Desenvolvimento Regional (FEDER) através do Programa Operacional (POCTEP).

OBJETIVOS: Caracterizar os hábitos alimentares, de saúde e a adesão ao padrão alimentar mediterrânico dos jovens do ensino secundário da região do Algarve.

METOdologiA: Estudo descritivo transversal onde se inquiriu, em ambiente escolar, uma amostra de jovens, do $10 .^{\circ}$ ano de escolaridade, dos cursos científico-humanísticos e cursos profissionais de oito escolas secundárias da região do Algarve. Recolheu-se informação através de um questionário de autopreenchimento com cinco secções: 1) hábitos alimentares; 2) adesão ao padrão alimentar mediterrânico (Índice KIDMED); 3) prática de atividade física; 4) higiene do sono e oral; 5) características sociodemográficas. Registou-se o peso, altura, perímetro da cintura e da anca, e a pressão arterial, obtidos através de metodologias de referência.

RESULTADOS: A amostra final incluiu 325 participantes, 47\% ( $n=153)$ do sexo masculino e 53\% ( $n=172)$ do sexo feminino, com idades entre 15 e 19 anos ( $M=16,4$ anos; $\mathrm{DP}=0,89$ anos). Analisou-se o percentil Índice de Massa Corporal/Idade de acordo com as recomendações da Organização Mundial da Saúde e registou-se uma prevalência de sobrecarga ponderal de 19,7\%. Os resultados do Índice KIDMED (M=6,9 pontos; DP=2,46) indicam uma baixa adesão ao padrão alimentar mediterrânico em 9,0\% dos participantes, adesão intermédia em $45,5 \%$ e adesão alta em 45,5\%.

CONCLUSÕES: Os resultados indicam que devem ser delineadas estratégias de educação alimentar e de promoção da saúde dirigidas à população juvenil, tendo por base a Dieta Mediterrânica.

\section{PALAVRAS-CHAVE}

Adolescentes, Dieta Mediterrânica, Excesso de peso, Hábitos alimentares, Obesidade, Saúde

ABSTRACT

INTRODUCTION: Study developed by the University of Algarve, under the Project 0290_MEDITA_5_P_"Mediterranean Diet Promotes Health", financed by the European Regional Development Fund (FEDER) through the operational programme (POCTEP). OBJECTIVES: Characterize the eating habits of health and adherence to the Mediterranean Dietary Pattern of young people of secondary school of the Algarve region.

METHODOLOGY: In a cross-sectional descriptive study, a sample of students, from the 10th grade, of the scientific-humanistic and professional courses from eight secondary schools in the Algarve region were surveyed. Information was collected through a self-filling questionnaire with five sections: 1) eating habits; 2) adherence to the Mediterranean Dietary Pattern (KIDMED Index); 3) practice of physical activity; 4) sleep and oral hygiene; 5) sociodemographic characteristics. Weight, height, waist and hip circumference, and blood pressure were recorded using reference methodologies.

RESULTS: The final sample included 325 participants, 47\% $(n=153)$ male and 53\% $(n=172)$ female, aged between 15 and 19 years ( $\mathrm{M}=16.4$ years; $\mathrm{DP}=0.89$ years). The Body Mass Index/Age percentile was measured according to World Health Organization recommendations and the prevalence of overweight was $19.7 \%$. The results of the KIDMED Index ( $M=6.9$ points, SD = 2.46) indicated a low adherence to the Mediterranean Dietary Pattern in 9,0\% of the participants, intermediate adherence in $45.5 \%$ and high adherence in $45.5 \%$.

CONCLUSIONS: The results indicate that strategies for food education and health promotion aimed at the youth population should be drawn upon the basis of the Mediterranean Diet

KEYWORDS

Adolescents, Mediterranean Diet, Overweight, Food habits, Obesity, Health 


\section{INTRODUÇÃO}

Os hábitos alimentares praticados durante a infância e a adolescência, nomeadamente o consumo de alimentos nutricionalmente pobres e de elevada densidade energética, podem tornar estas fases particularmente vulneráveis a situações de obesidade e carências nutricionais específicas $(1,2)$. Maus hábitos alimentares e estilos de vida sedentários, quando desenvolvidos na primeira infância e especialmente na adolescência, tendem a ser reforçados e mantidos na vida adulta (3).

Uma dieta saudável sustentável e um estilo de vida ativo podem limitar os comportamentos prejudiciais que contribuem para a epidemia de doenças crónicas não transmissíveis, como a diabetes, hipertensão, doenças cardiovasculares, apneia do sono e certos tipos de cancro (4). Conhecer os hábitos de vida dos adolescentes é condição determinante para poder intervir adequadamente em estratégias preventivas, focadas na promoção da saúde, através da ação articulada de fatores relacionados com estilos de vida, ao nível dos hábitos alimentares e prática de atividade física (5).

A Dieta Mediterrânica (DM) é considerada, pela Organização Mundial da Saúde (OMS), como um importante modelo salutogénico, com efeito no controlo do peso, prevenção de doenças crónicas e aumento da longevidade, pelo que a sua promoção torna-se fundamental junto dos adolescentes $(6,7)$. Investir na sua saúde traz vantagens na saúde imediata e futura e na saúde da sua descendência (4).

O Algarve, pelas suas características geográficas e socioculturais, é uma região onde se espera que as tradições alimentares mediterrânicas estejam presentes. Contudo, não existem publicações na literatura científica que permitam identificar o padrão alimentar e a adesão à DM em adolescentes algarvios. De maneira a caracterizar os hábitos alimentares de adolescentes da região e obter informação que possa servir de base para intervenção alimentar, submeteu-se uma candidatura ao Fundo Europeu de Desenvolvimento Regional (FEDER) através do Programa Operacional (POCTEP) para desenvolver o Projeto 0290_MEDITA_5_P - "Dieta Mediterrânica Promove Saúde". Esta candidatura foi aceite e este artigo apresenta os resultados da primeira fase do projeto.

\section{OBJETIVOS}

Caracterizar os hábitos alimentares, de saúde e a adesão ao padrão alimentar mediterrânico de jovens da região do Algarve.

\section{METODOLOGIA}

Estudo descritivo transversal, aprovado pela Comissão Nacional de Proteção de Dados (Autorização n. ${ }^{\circ}$ 234/2018) e Direção-Geral de Inovação e Desenvolvimento Curricular (Registro n. ${ }^{\circ} 0625700001$ ).

\section{População-alvo e amostra}

De acordo com os recursos alocados ao projeto, tempo de execução e natureza deste estudo, direcionou-se as atividades da primeira fase apenas a estudantes do $10 .^{\circ}$ ano de escolaridade. Foi selecionada uma amostra aleatória estratificada, multietapas, representativa dos alunos do $10 .^{\circ}$ ano dos cursos científico-humanísticos e cursos profissionais do Ensino Secundário, na região do Algarve. De acordo com o método proposto por Fleiss e colaboradores (8), estimou-se que devia inquirir-se oito das dezasseis escolas secundárias da região, perfazendo uma amostra final de 545 jovens. A estratificação foi levada a cabo tendo em conta a densidade estudantil. De maneira a aumentar a validade e fiabilidade da inquirição, definiram-se como fatores de exclusão: 1) gravidez; 2) incapacidade física e/ou doença mental. As participantes grávidas poderiam enviesar os resultados das avaliações antropométricas e poderiam ter um padrão alimentar específico, diferente dos restantes elementos da população-alvo. Participantes que apresentassem incapacidade física e/ou doença mental poderiam inviabilizar as avaliações antropométricas ou terem a capacidade de compreensão do questionário comprometida.

Participaram neste estudo apenas os jovens autorizados pelos encarregados de educação, mediante consentimento informado, livre e esclarecido, e que, simultaneamente, manifestaram vontade em participar.

Foi desenvolvido o questionário "Alimentação, estilo de vida e saúde", de autopreenchimento, com cinco secções: 1) hábitos alimentares; 2) adesão ao Padrão Alimentar Mediterrânico (Índice KIDMED); 3) prática de atividade física; 4) hábitos de saúde (higiene do sono e oral); e 5) características sociodemográficas.

A adesão ao Padrão Alimentar Mediterrânico (PAM) foi estudada a partir da versão portuguesa do índice KIDMED utilizada no estudo de Mateus MP (9). Na tradução portuguesa, a autora optou por redigir a questão relativa ao pequeno-almoço como "Costumas tomar o pequeno-almoço?", na forma positiva, e com isso ajustou a pontuação, em que a resposta "sim" passa a corresponder a "0" e o "não" corresponde a "-1".

Criou-se um protocolo para a avaliação física, com registo do peso, altura, perímetro da cintura, perímetro da anca e da pressão arterial, recolhidos através de metodologias de referência e cálculo do Índice de Massa Corporal (IMC) $(10,11)$. Utilizou-se equipamento validado e certificado para o efeito: balança digital Seca $877^{\circledR}$; estadiómetro portátil Seca $217^{\oplus}$; fita métrica de teflon flexível e indeformável; e esfigmomanómetro Medel Elite ${ }^{\circledR}$. A classificação do estado nutricional teve por base o percentil IMC/Idade, segundo os critérios da OMS que utiliza as curvas de crescimento para crianças dos 5 aos 19 anos (12). A classificação da hipertensão foi feita com recurso às guidelines da Sociedade Europeia de Hipertensão para crianças e adolescentes (13).

\section{Análise estatística}

Utilizou-se o software IBM-SPSS ${ }^{\circledR}$ versão 25.0 (SPSS Inc., Chicago, IL, USA) para a análise estatística.

Para comparações entre dois grupos utilizou-se o teste t-de-Student ou o teste de Mann-Whitney, para comparações de múltiplos grupos utilizou-se o teste de Kruskal-Wallis ou a análise de variância (ANOVA), juntamente com a correção de Bonferroni sempre que necessário. Utilizou-se o coeficiente de correlação de Pearson para analisar a associação entre variáveis com distribuição Normal. Em todas as outras situações onde se levou a cabo um teste de correlação foi interpretado o coeficiente de correlação de Spearman.

Utilizaram-se os testes de independência e de homogeneidade do qui-quadrado para estudar variáveis categóricas. Em situações onde a aplicação do teste era limitada pelo número de respostas, optou-se por acompanhar o teste por um procedimento de bootstrap, biascorrected (BCa) com 1000 amostras aleatórias.

Analisou-se o IMC de acordo com os critérios da OMS (12) e calcularam-se os respetivos z-scores através do software WHO Anthro (versão 3.2.2, January 2011).

Considerou-se, em todos os testes, um intervalo de significância inferior a 0,05 como indicação de significado estatístico.

\section{RESULTADOS}

\section{Caracterização sociodemográfica}

Registou-se uma taxa de resposta de 59,6\%, correspondendo a uma amostra final composta por 325 participantes, 47\% ( $n=153)$ do sexo masculino e 53\% ( $n=172)$ do sexo feminino, com idades entre os quinze e os dezanove anos ( $M=16,4$ anos; $D P=0,89$ anos). 
No geral, os participantes vivem num meio não rural $(78,5 \%, n=255)$, são de nacionalidade portuguesa $(88,6 \% ; n=287)$ e naturais do Algarve (76,3\%; $n=244)$. Relativamente à composição dos agregados familiares, a maior parte $(67,0 \%$; $n=217)$ vivem com a mãe e com o pai, com maior prevalência da nacionalidade portuguesa em ambos os progenitores (MÃE: 80,4\%; n=230; PAI: 78,9\%; n=183).

\section{Hábitos alimentares}

Mais de um terço dos participantes $(41 \% ; n=134)$ referiram ter modificado os seus hábitos alimentares nos últimos 12 meses, e os três principais motivos foram: "Preocupações do próprio com a imagem ou com o peso" (22,4\%; n=30), "Preocupação do próprio com a qualidade da alimentação" (23,1\%; n=31), e "Motivos de saúde" (26,1\%; n=35). Cerca de $7 \%(n=24)$ refere seguir um padrão alimentar especial, do qual a exclusão da lactose (66,7\%; n=16), da carne (29,2\%; $n=7)$ e do glúten (20,8\%; n=5) são as alterações predominantes. A maior parte dos jovens inquiridos faz entre quatro e seis refeições diárias, com uma média de cinco refeições $(\mathrm{DP}= \pm 0,95)$. A merenda da manhã $(28,6 \%$; $n=93)$ e a ceia $(59,7 \%$ ( $n=194)$ são as refeições menos realizadas. Verificou-se também que 13,2\% ( $n=43$ ) não toma regularmente o pequeno-almoço, por "falta de tempo" $(32,6 \%$; $n=14)$ ou "falta de apetite" (39,5\%; $n=17)$ como os motivos mais referidos.

Os participantes fazem, em média, 1,62 refeições intermédias ( $\mathrm{DP}= \pm 0,57$ ). Verificou-se que 4,6\% ( $n=15)$ referem não fazer nenhuma, 28,3\% (n=92) fazem apenas uma e 67,1\% ( $n=218$ ) fazem duas refeições intermédias ao longo do dia. A Tabela 1 mostra a informação relativa ao pequeno-almoço e merendas dos participantes, no que respeita ao local, companhia e alimentos mais consumidos. De referir que se encontrou um consumo de alimentos de elevada densidade energética nestas refeições (Tabela 2). Obtiveram-se diferenças estatisticamente significativas entre sexos para o pequeno-almoço $\left(X^{2}=4,193 ; p=0,041\right)$ e para a merenda da manhã $\left(X^{2}=7,609 ; p=0,006\right)$, verificando-se que os rapazes referem uma maior frequência de consumo destas refeições comparativamente às raparigas. Também foi possível identificar diferenças estatisticamente significativas entre sexos no consumo de alguns alimentos. Os rapazes referem mais o consumo de leite $(p=0,003)$, mas as raparigas referem mais o consumo de fruta $(p=0,024)$, frutos secos e oleaginosos $(p=0,028)$, sumos de fruta $100 \%$ ( $p=0,007)$ e chá ou infusões ( $p<0,001)$.

Encontrou-se uma associação positiva estatisticamente significativa entre a toma do pequeno-almoço e o número de refeições em família ( $M W=4519,500 ; p=0,004)$.

Todos os participantes referem almoçar ( $n=325)$, contudo 1,2\% $(n=4)$ destes não jantam (Tabela 3). Foi possível observar que cerca de 31\% $(n=100)$ dos participantes comem sandes em substituição do prato ao almoço e 9,3\% (n=30) ao jantar, e que mais de metade refere fazer um consumo habitual de leguminosas ao almoço $(58,2 \%$; $n=173)$ e ao jantar (55,6\%; n=163).

O café é consumido por 23,3\% ( $n=67)$ dos participantes ao almoço e por 12,9\% ( $n=36)$ ao jantar. Obtiveram-se diferenças estatisticamente significativas entre sexos para o consumo de café ao jantar $\left(X^{2}=9,269\right.$; $p=0,002)$, verificando-se que os rapazes referem um maior consumo do que as raparigas. Relativamente à ingestão de água ao longo do dia, 37,5\% ( $n=122)$ dos participantes refere consumir entre 0,5L e 1L. No que diz respeito ao consumo de bebidas alcoólicas, a maior parte dos participantes raramente ou nunca consomem bebidas alcoólicas (80,6\%, n=262). Contudo, 23,7\% ( $n=78)$ dos jovens inquiridos consome bebidas destiladas (puras, com sumo ou refrigerantes e em shots), 23,1\% ( $n=75$ ) consome cidra ou cerveja com sabores ou com refrigerantes, 16,6\% ( $n=54)$ consome cerveja e 5,8\% $(n=19)$ reporta o consumo de vinho. Encontraram-se diferenças estatisticamente significativas entre sexos para o consumo de cerveja $\left(X^{2}=19,5\right.$; $p<0,001$ ), com os participantes do sexo masculino a reportarem um consumo mais frequente desta bebida alcoólica.

\section{Adesão ao padrão alimentar mediterrânico}

Os resultados do Índice KIDMED apresentam uma pontuação média de 6,9 pontos ( $\mathrm{DP}=2,46)$ e mostram uma baixa adesão ao PAM em 9,0\% dos jovens, uma adesão intermédia em 45,5\% e uma alta adesão em $45,5 \%$. Os dados relativos às respostas "sim" de cada pergunta do Índice KIDMED encontram-se na Tabela 4. Foi possível identificar diferenças estatisticamente significativas entre sexos para algumas perguntas. Assim, os rapazes referem mais vezes "frequentar, mais de uma vez por semana, restaurantes de fast-food" ( $(\mathrm{p}=0,006)$, "consumir massa ou arroz, quase todos os dias (5 ou mais vezes por semana)" ( $p<0,001)$.

Um maior número de refeições diárias associou-se significativamente a uma maior adesão ao PAM ( $\left.r_{\text {spearman }}=0,195 ; p<0,001\right)$, bem como a toma do pequeno-almoço ( $M W=2408,500 ; p<0,001)$. Também o número de refeições em família se associou significativamente a uma maior adesão ao PAM $\left(r_{\text {spearman }}=0,161 ; p=0,004\right)$. Os alunos que consomem habitualmente sopa ao almoço apresentaram uma maior adesão ao PAM ( $M W=8843,500 ; p<0,001)$ e ao jantar também ( $M W=7190,000 ; p<0,001)$.

\section{Avaliação física}

Relativamente ao estado nutricional analisado a partir do Percentil IMC/ Idade, verifica-se que 19,7\% dos participantes encontram-se com sobrecarga ponderal, dos quais, 17,2\% ( $n=56)$ com excesso de peso e 2,5\% ( $n=8$ ) com obesidade (Tabela 5). Não se verificaram diferenças estatisticamente significativas entre sexos nesta variável $\left(X^{2}=1,35\right.$, $p=0,718)$. Observou-se que $2,2 \%(n=7)$ dos alunos apresentaram hipertensão e que 10,2\% (n=33) apresentaram valores de pressão arterial normal-alta (Tabela 5). A Tabela 6 resume as características antropométricas e o valor da pressão arterial dos participantes. Verificaram-se diferenças estatisticamente significativas entre sexos $(p<0,05)$ no peso, altura, perímetro da cintura, perímetro da anca e na pressão arterial sistólica. Não se encontraram diferenças estatisticamente significativas na associação das categorias de hipertensão com o nível de adesão ao PAM.

O número de refeições associou-se de forma negativa e estatisticamente significativa ao percentil IMC/ldade $\left(r_{\text {spearman }}=-0,152\right.$; $\mathrm{p}=0,006$ ), ou seja, quanto maior o número de refeições diárias, menor o percentil IMC/Idade. Não se encontrou associação estatisticamente significativa entre a toma do pequeno-almoço e o percentil IMC/Idade ( $M W=5113,500 ; p=0,098)$, contudo encontrou-se com o perímetro da cintura ( $M W=4898,500 ; p=0,046)$, sendo que quem não toma o pequeno-almoço apresenta um perímetro da cintura mais elevado. Ao relacionar a adesão ao PAM com elementos da avaliação física não se encontraram diferenças estatisticamente significativas para o percentil IMC/Idade $\left(r_{\text {speaman }}=0,061 ; p=0,275\right)$, nem para o perímetro da cintura $\left(r_{\text {spearman }}=0,030 ; p=0,591\right)$, nem para os níveis de pressão arterial $\left(r_{\text {spearman }}=-0,018 ; p=0,749\right)$.

O perímetro da cintura associou-se positivamente e de forma significativa ao percentil IMC/Idade $\left(r_{\text {spearman }}=0,739 ; p<0,001\right)$, bem como a pressão arterial $(r$ $=0,225 ; p<0,001)$.

\section{Atividade física e hábitos de saúde}

No que diz respeito à atividade física, 90,2\% ( $n=293)$ dos participantes referem não praticar desporto escolar. Dos que o fazem, a média de horas semanal mais referida, foi de 2 horas $(31,3 \%, n=10)$. Verificaram-se diferenças estatisticamente significativas no total de tempo dedicado ao desporto escolar durante a semana $\left(X^{2}=10,400 ; p=0,034\right)$, verificando-se 
que os rapazes dedicam mais tempo que as raparigas.

Mais de metade dos jovens inquiridos $(55,4 \%, n=180)$ pratica desporto fora da escola, e destes $42,5 \%$ ( $n=76)$ dedicam, uma média, de mais de 5 horas semanais, a essa prática. Encontraram-se diferenças estatisticamente significativas entre sexos, com os rapazes a praticar mais desporto fora da escola do que as raparigas $\left(X^{2}=20,518\right.$; $p<0,001)$ e a dedicar mais tempo semanal a esses desportos $\left(X^{2}=12,960 ; p=0,011\right)$. Não se encontraram diferenças estatisticamente significativas no percentil IMC/Idade ( $K W=4899,500 ; p=0,042)$, na pressão arterial ( $K W=9,683 ; p=0,021)$ nem no perímetro da cintura (KW=12547,000; $p=0,608$ ) entre os praticantes e não praticantes de desporto fora da escola.

\section{Tabela 1}

Informação relativa ao pequeno-almoço, meio da manhã e da tarde

\begin{tabular}{|c|c|c|c|c|c|c|c|c|}
\hline & & & \multicolumn{2}{|c|}{ TOTAL } & \multicolumn{2}{|c|}{ FEMININO } & \multicolumn{2}{|c|}{ MASCULINO } \\
\hline & & & $\%$ & $\mathrm{n}$ & $\%$ & $\mathbf{N}$ & $\%$ & $\mathrm{n}$ \\
\hline \multirow{12}{*}{$\begin{array}{l}\text { Pequeno-almoço } \\
(n=282)\end{array}$} & \multirow{4}{*}{ Local } & Casa & 92,2 & 260 & 92,3 & 132 & 92,1 & 128 \\
\hline & & Escola & 6,0 & 17 & 5,6 & 8 & 6,5 & 9 \\
\hline & & Pastelaria & 0,7 & 2 & 0,7 & 1 & 0,7 & 1 \\
\hline & & Outra & 1,1 & 3 & 1,4 & 2 & 0,7 & 1 \\
\hline & \multirow{3}{*}{ Com quem } & Família & 48,9 & 138 & 46,2 & 66 & 51,8 & 72 \\
\hline & & Amigos/colegas & 6,0 & 17 & 6,3 & 9 & 5,8 & 8 \\
\hline & & Sozinho (a) & 45,0 & 127 & 47,6 & 68 & 42,4 & 59 \\
\hline & \multirow{5}{*}{$\begin{array}{l}\text { Alimentos mais } \\
\text { consumidos }\end{array}$} & Pão & 75,2 & 212 & 73,4 & 105 & 77,0 & 107 \\
\hline & & Leite & 74,8 & 211 & 67,1 & 96 & 82,7 & 115 \\
\hline & & Cereais & 68,4 & 193 & 65,0 & 93 & 71,9 & 100 \\
\hline & & Manteiga, margarina & 56,4 & 159 & 56,6 & 81 & 56,1 & 78 \\
\hline & & Fruta & 54,3 & 153 & 60,8 & 87 & 47,5 & 66 \\
\hline \multirow{13}{*}{$\begin{array}{l}\text { Meio da manhã } \\
(n=232)\end{array}$} & \multirow{4}{*}{ Local } & Casa & 2,2 & 5 & 0,7 & 1 & 4,1 & 4 \\
\hline & & Escola & 90,9 & 211 & 91,8 & 123 & 89,8 & 88 \\
\hline & & Pastelaria & 6,5 & 15 & 7,5 & 10 & 5,1 & 5 \\
\hline & & Outra & 0,4 & 1 & 0,0 & 0 & 1,0 & 1 \\
\hline & \multirow{3}{*}{ Com quem } & Família & 1,3 & 3 & 0,7 & 1 & 2,0 & 2 \\
\hline & & Amigos/colegas & 90,1 & 209 & 93,3 & 125 & 85,7 & 84 \\
\hline & & Sozinho (a) & 8,6 & 20 & 6,0 & 8 & 12,2 & 12 \\
\hline & \multirow{6}{*}{$\begin{array}{l}\text { Alimentos mais } \\
\text { consumidos }\end{array}$} & Pão & 70,7 & 164 & 70,1 & 94 & 71,4 & 70 \\
\hline & & Bolos, bolachas & 55,6 & 129 & 57,5 & 77 & 53,1 & 52 \\
\hline & & $\begin{array}{l}\text { Pão de leite, croissant, pão } \\
\text { de Deus }\end{array}$ & 44,8 & 104 & 48,5 & 65 & 39,8 & 39 \\
\hline & & Produtos de charcutaria & 44,8 & 104 & 46,3 & 62 & 42,9 & 42 \\
\hline & & Manteiga, margarina & 42,7 & 99 & 41,0 & 55 & 44,9 & 44 \\
\hline & & Fruta & 41,6 & 96 & 47,4 & 63 & 33,7 & 33 \\
\hline \multirow{13}{*}{$\begin{array}{l}\text { Lanche } \\
(n=296)\end{array}$} & \multirow{4}{*}{ Local } & Casa & 70,3 & 208 & 68,8 & 110 & 72,1 & 98 \\
\hline & & Escola & 26,0 & 77 & 28,1 & 45 & 23,5 & 32 \\
\hline & & Pastelaria & 2,4 & 7 & 2,5 & 4 & 2,2 & 3 \\
\hline & & Outra & 1,4 & 4 & 0,6 & 1 & 2,2 & 3 \\
\hline & \multirow{4}{*}{ Com quem } & Família & 19,6 & 58 & 18,8 & 30 & 20,6 & 28 \\
\hline & & Amigos/colegas & 25,7 & 76 & 27,5 & 44 & 23,5 & 32 \\
\hline & & Sozinho (a) & 54,1 & 160 & 52,5 & 84 & 55,9 & 76 \\
\hline & & Outra & 0,7 & 2 & 1,3 & 2 & 0,0 & 0 \\
\hline & \multirow{5}{*}{$\begin{array}{l}\text { Alimentos mais } \\
\text { consumidos }\end{array}$} & Pão & 81,4 & 241 & 80,0 & 128 & 83,1 & 113 \\
\hline & & Fruta & 59,5 & 176 & 65,6 & 105 & 52,2 & 71 \\
\hline & & Manteiga, margarina & 55,7 & 165 & 55,6 & 89 & 55,9 & 76 \\
\hline & & Bolos, bolachas & 54,7 & 162 & 55,6 & 89 & 53,7 & 73 \\
\hline & & Produtos de charcutaria & 53,0 & 157 & 56,3 & 90 & 49,3 & 67 \\
\hline
\end{tabular}

Tabela 2

Alimentos de elevada densidade energética consumidos às refeições

\begin{tabular}{|c|c|c|c|c|c|c|c|c|}
\hline & \multicolumn{2}{|c|}{ PEQUENO-ALMOÇO } & \multicolumn{2}{|c|}{ MEIO DA MANHÃ } & \multicolumn{2}{|c|}{ LANCHE } & \multicolumn{2}{|c|}{ CEIA } \\
\hline & $\%$ & $\mathrm{n}$ & $\%$ & $\mathrm{n}$ & $\%$ & $\mathrm{~N}$ & $\%$ & $\mathrm{n}$ \\
\hline Pão de leite/croissant/pão de Deus & 24,4 & 69 & 44,8 & 104 & 37,8 & 112 & 18,3 & 24 \\
\hline Bolos/bolachas & 18,4 & 52 & 55,6 & 129 & 54,7 & 162 & 50,4 & 66 \\
\hline Snacks doces & 6,4 & 18 & 23,3 & 54 & 28,7 & 85 & 26,7 & 35 \\
\hline Snacks salgados & 5,3 & 15 & 7,8 & 18 & 13,5 & 40 & 12,2 & 16 \\
\hline Refrigerantes & 7,1 & 20 & 14,2 & 33 & 22,3 & 66 & 16,0 & 21 \\
\hline
\end{tabular}


Tabela 3

Informação relativa ao almoço e ao jantar

\begin{tabular}{|c|c|c|c|c|c|c|c|c|}
\hline & & & \multicolumn{2}{|c|}{ TOTAL } & \multicolumn{2}{|c|}{ FEMININO } & \multicolumn{2}{|c|}{ MASCULINO } \\
\hline & & & $\%$ & $\mathrm{n}$ & $\%$ & $\mathrm{~N}$ & $\%$ & $\mathrm{n}$ \\
\hline \multirow{18}{*}{$\begin{array}{l}\text { Almoço } \\
(n=325)\end{array}$} & \multirow{5}{*}{ Local } & Casa & 32,9 & 107 & 38,4 & 66 & 26,8 & 41 \\
\hline & & Cantina & 41,5 & 135 & 43,0 & 74 & 39,9 & 61 \\
\hline & & Bufete & 5,5 & 18 & 2,3 & 4 & 9,2 & 14 \\
\hline & & Snack-bar/restaurante & 16,9 & 55 & 15,1 & 26 & 19,0 & 29 \\
\hline & & Outra (não especificaram) & 3,1 & 10 & 1,2 & 2 & 5,2 & 8 \\
\hline & \multirow{4}{*}{ Com quem } & Família & 22,2 & 72 & 23,3 & 40 & 20,9 & 32 \\
\hline & & Amigos/colegas & 64,6 & 210 & 61,0 & 105 & 68,6 & 105 \\
\hline & & Sozinho (a) & 12,0 & 39 & 14,5 & 25 & 9,2 & 14 \\
\hline & & Outra (não especificaram) & 1,2 & 4 & 1,2 & 2 & 1,3 & 2 \\
\hline & \multirow{9}{*}{ Alimentos } & Sopa $(n=325)$ & 48,9 & 159 & 54,1 & 93 & 43,1 & 66 \\
\hline & & Salada $(n=297)$ & 78,5 & 234 & 81,9 & 131 & 74,6 & 103 \\
\hline & & Sobremesa $(n=325)$ & 60,3 & 196 & 55,2 & 95 & 66,0 & 101 \\
\hline & & Fruta & 84,2 & 165 & 89,5 & 85 & 79,2 & 80 \\
\hline & & Doce & 15,8 & 31 & 10,5 & 10 & 20,8 & 21 \\
\hline & & Bebida $(n=325)$ & 88,6 & 288 & 86,0 & 148 & 91,5 & 140 \\
\hline & & Água & 96,9 & 279 & 97,3 & 144 & 96,4 & 135 \\
\hline & & Refrigerantes & 45,5 & 131 & 39,2 & 58 & 52,1 & 73 \\
\hline & & Néctares de fruta & 43,4 & 125 & 37,8 & 56 & 49,3 & 69 \\
\hline \multirow{16}{*}{$\begin{array}{l}\text { Jantar } \\
(n=321)\end{array}$} & \multirow{3}{*}{ Local } & Casa & 99,4 & 319 & 100,0 & 169 & 98,7 & 150 \\
\hline & & Snack-bar/restaurante & 0,3 & 1 & 0,0 & 0 & 0,7 & 1 \\
\hline & & Outra & 0,3 & 1 & 0,0 & 0 & 0,7 & 1 \\
\hline & \multirow{4}{*}{ Com quem } & Família & 94,1 & 302 & 94,1 & 159 & 94,1 & 143 \\
\hline & & Amigos/colegas & 0,0 & 0 & 0,0 & 0 & 0,0 & 0 \\
\hline & & Sozinho (a) & 5,6 & 18 & 5,3 & 9 & 5,9 & 9 \\
\hline & & Outra & 0,3 & 1 & 0,6 & 1 & 0,0 & 0 \\
\hline & \multirow{9}{*}{ Alimentos } & Sopa $(n=321)$ & 48,6 & 156 & 51,5 & 87 & 45,4 & 69 \\
\hline & & Salada (n=293) & 79,5 & 233 & 84,0 & 131 & 74,5 & 102 \\
\hline & & Sobremesa $(n=321)$ & 62,9 & 202 & 61,5 & 104 & 64,5 & 98 \\
\hline & & Fruta & 77,5 & 155 & 80,8 & 84 & 74,0 & 71 \\
\hline & & Doce & 22,5 & 45 & 19,2 & 20 & 26,0 & 25 \\
\hline & & Bebida $(n=321)$ & 86,6 & 278 & 84,6 & 143 & 88,8 & 135 \\
\hline & & Água & 95,0 & 264 & 95,1 & 136 & 94,8 & 128 \\
\hline & & Refrigerantes & 41,7 & 116 & 35,7 & 51 & 48,1 & 65 \\
\hline & & Néctares de fruta & 38,1 & 106 & 32,2 & 46 & 44,4 & 60 \\
\hline
\end{tabular}

\section{Tabela 4}

Respostas ao Índice KIDMED dos participantes

\begin{tabular}{|c|c|c|c|}
\hline & PREVALÊNCIA (\%) & IC $95 \%$ & $\mathbf{N}$ \\
\hline Consomes uma peça de fruta ou sumo de fruta todos os dias? & 68,6 & $63,4-73,5$ & 223 \\
\hline Consomes uma segunda peça de fruta todos os dias? & 37,8 & $32,7-43,2$ & 123 \\
\hline Consomes regularmente produtos hortícolas frescos ou cozinhados, pelo menos uma vez por dia? & 68,9 & $63,7-73,8$ & 224 \\
\hline Consomes regularmente produtos hortícolas frescos ou cozinhados, mais de uma vez por dia? & 43,4 & $38,1-48,8$ & 141 \\
\hline Consomes regularmente peixe (pelo menos, 2 a 3 vezes por semana)? & 64,3 & $59-69,4$ & 209 \\
\hline Frequentas, mais de uma vez por semana, restaurantes de fast-food? & 15,1 & $11,5-13,3$ & 49 \\
\hline Consomes leguminosas, mais de uma vez por semana? & 64,3 & $59-69,4$ & 209 \\
\hline Consomes massa ou arroz, quase todos os dias ( 5 ou mais vezes por semana)? & 72,9 & $67,9-77,5$ & 237 \\
\hline Consomes cereais ou produtos derivados de cereais (pão, etc.) ao pequeno-almoço? & 83,7 & $79,4-87,4$ & 272 \\
\hline $\begin{array}{l}\text { Consomes regularmente frutos oleaginosos (nozes, amêndoas, etc.), pelo menos } 2 \text { a } 3 \text { vezes por } \\
\text { semana? }\end{array}$ & 29,8 & $25,1-35$ & 97 \\
\hline Usas azeite em casa? & 94,8 & $91,9-96,8$ & 308 \\
\hline Costumas tomar o pequeno-almoço? & 87,7 & $83,8-90,9$ & 285 \\
\hline Consomes laticínios (leite, iogurte, etc.) ao pequeno-almoço? & 82,8 & $78,4-86,6$ & 269 \\
\hline Consomes produtos confecionados ou de pastelaria ao pequeno-almoço? & 22,2 & $17,9-26,9$ & 72 \\
\hline Consomes diariamente 2 iogurtes e/ou queijo $(40 \mathrm{~g}) ?$ & 40,0 & $34,8-45,4$ & 130 \\
\hline Consomes doces ou guloseimas todos os dias? & 16,3 & $12,6-20,6$ & 53 \\
\hline
\end{tabular}

Apresentadas as respostas "sim" para cada uma das perguntas. 
Tabela 5

Categorias do Percentil IMC/Idade e da Pressão Arterial dos participantes

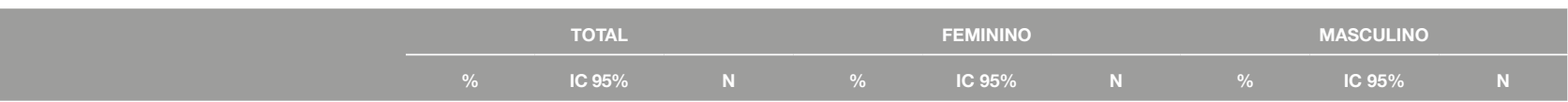

Categorias do Percentil IMC/Idade

Total $(\mathrm{N}=325)$ Feminino $(\mathrm{N}=172)$ Masculino $(\mathrm{N}=153)$

\begin{tabular}{|c|c|c|c|c|c|c|c|c|c|}
\hline Magreza & 0,9 & $0,3-2,4$ & 3 & 0,6 & $0,0-2,7$ & 1 & 1,3 & $0,3-4,1$ & 2 \\
\hline Peso normal & 79,4 & $74,7-83,5$ & 258 & 80,8 & $74,4-86,2$ & 139 & 77,8 & $70,7-83,8$ & 119 \\
\hline Excesso de peso & 17,2 & $13,4-21,6$ & 56 & 16,9 & $11,8-23,0$ & 29 & 17,6 & $12,2-24,3$ & 27 \\
\hline Obesidade & 2,5 & $1,2-4,6$ & 8 & 1,7 & $0,5-4,6$ & 3 & 3,3 & $1,3-7,0$ & 5 \\
\hline \multicolumn{10}{|c|}{$\begin{array}{l}\text { Categorias de Pressão Arterial } \\
\text { Total }(N=323) \text { Feminino ( } N=170) \text { Masculino }(N=153)\end{array}$} \\
\hline Pressão arterial normal & 84,8 & $80,6-88,4$ & 274 & 87,6 & $82,1-91,9$ & 149 & 81,7 & $75,0-87,2$ & 125 \\
\hline Pressão arterial normal-alta & 10,2 & $7,3-13,9$ & 33 & 8,2 & $4,8-13,1$ & 14 & 12,4 & $7,9-18,3$ & 19 \\
\hline Hipertensão sistólica isolada & 2,8 & $1,4-5,0$ & 9 & 2,4 & $0,8-5,5$ & 4 & 3,3 & $1,3-7,0$ & 5 \\
\hline Hipertensão & 2,2 & $1,0-4,2$ & 7 & 1,8 & $0,5-4,6$ & 3 & 2,6 & $0,9-6,1$ & 4 \\
\hline
\end{tabular}

IC: Intervalo de confiança

IMC: Índice de Massa Corporal

\section{Tabela 6}

Características antropométricas e de pressão arterial dos participantes

\begin{tabular}{|c|c|c|c|c|c|c|c|c|}
\hline & \multicolumn{2}{|c|}{ TOTAL } & \multicolumn{3}{|c|}{ FEMININO } & \multicolumn{3}{|c|}{ MASCULINO } \\
\hline & M & DP & M & MÍN - MÁX & DP & M & MÍN - MÁX & DP \\
\hline Peso (kg) & 60,3 & 11,1 & 56,7 & $42,0-88,8$ & 9,9 & 64,3 & $47,5-111,5$ & 11,1 \\
\hline Altura (m) & 1,66 & 0,086 & 1,61 & $1,45-1,83$ & 0,062 & 1,72 & $1,53-1,92$ & 0,067 \\
\hline Percentil IMC/Idade & 54 & 27,6 & 56,3 & $1,5-98,5$ & 26,2 & 51,5 & $0,6-99,2$ & 28,9 \\
\hline P. da cintura $(\mathrm{cm})$ & 71,7 & 8,7 & 70,0 & $56,0-99,0$ & 7,8 & 73,6 & $58-112$ & 9,2 \\
\hline P. da anca $(\mathrm{cm})$ & 93,9 & 7,7 & 94,7 & $62,5-116,5$ & 7,9 & 92,9 & $78-115$ & 7,4 \\
\hline PA sistólica (mm Hg) & 117 & 12 & 114 & $80-149$ & 12 & 120 & $87-176$ & 12 \\
\hline PA diastólica $(\mathrm{mm} \mathrm{Hg})$ & 70 & 9 & 71 & $38-94$ & 8 & 70 & $31-102$ & 9 \\
\hline
\end{tabular}

DP: Desvio-padrão

IMC: Índice de Massa Corpora

M: Média

Relativamente às horas durante a semana que os participantes passam a ver televisão ou a jogar videojogos, verificou-se que $27,8 \%$ ( $n=90)$, dedica uma média, de 2 horas por dia, e que durante o fim de semana essa média sobe para mais de 5 horas em $27,9 \%(n=90)$ dos participantes.

Registou-se uma média de 8 horas de sono durante a semana e uma média de 9,2 horas de sono durante o fim-de-semana. Verificaram-se diferenças na duração média do sono entre os dias da semana e os dias de fim-de-semana (teste de Wilcoxon; $p<0,001$ ). De destacar que durante a semana, 37,8\% dos alunos refere dormir menos de 8 horas. Encontrou-se uma associação negativa estatisticamente significativa entre o número de horas sedentárias semanais e o nível de adesão ao PAM $\left(r_{\text {spearman }}=-0,175 ; p=0,002\right)$. A prática de desporto fora da escola ( $M W=9548,000 ; p<0,001$ ) e de atividades desportivas de lazer ( $M W=8560,000 ; p<0,001)$ associaram-se de forma significativa com uma maior adesão ao PAM, assim como os participantes que bebem uma maior quantidade de água por dia ( $K W=28,572 ; p<0,001)$.

Um maior número de horas de sono semanais associou-se positiva e significativamente com uma maior adesão ao PAM $\left(r_{\text {spearman }}=0,142\right.$; $\mathrm{p}=0,010$ ).

Verificaram-se diferenças estatisticamente significativas na quantidade de água ingerida ao longo do dia, de acordo com número de horas de atividade física semanal $\left(r_{\text {spearman }}=0,298 ; p<0,001\right)$, ou seja, quem pratica mais horas de atividade física semanal consome uma quantidade de água superior. Também se encontraram diferenças estatisticamente significativas no número de refeições feitas pelos alunos que praticam desporto fora da escola ( $M W=11057,500 ; p=0,012$ ), apresentando
Mín - Máx: Valor mínimo e máximo

P: Perímetro

PA: Pressão Arterial

estes, uma média de refeições maior.

Assim como, entre a toma do pequeno-almoço e o número de horas de sono duranta a semana ( $M W=4899,500 ; p=0,042)$, em que os participantes que tomam o pequeno-almoço dormem mais horas comparativamente aos que não o tomam.

\section{DISCUSSÃO DOS RESULTADOS}

Os resultados obtidos indicam que, no geral, os adolescentes da região possuem hábitos alimentares que se distanciam de algumas das principais recomendações. A literatura descreve a importância do pequeno-almoço para o rendimento intelectual (14), mas cerca de $13 \%$ dos participantes deste trabalho não tomam habitualmente o pequeno-almoço, uma prevalência mais elevada do que a registada em adolescentes a nível nacional (15). Apesar de nesta refeição o leite ser um dos cinco alimentos mais consumidos $(74,8 \%$ dos participantes referem o seu consumo habitual), este assume uma importância menor em refeições subsequentes, não havendo menção a este alimento ou a qualquer um dos seus derivados nos cinco alimentos mais consumidos, por exemplo, na refeição a meio da manhã ou no lanche. A literatura sugere que existe uma associação negativa entre a ingestão de leite, iogurte e bebidas à base de leite e iogurte, e o excesso de gordura total e abdominal (16), pelo que estes resultados indicam que a promoção dos produtos lácteos nas refeições intermédias pode constituir um campo de atuação para a melhoria dos hábitos alimentares.

Ainda que os participantes identifiquem a fruta como um dos alimentos 
mais consumidos, as suas respostas no índice KIDMED contradizem o que declararam anteriormente, pois verificou-se que 31,4\% ( $n=102)$ dos jovens não consome fruta diariamente. Contudo, este resultado é superior ao encontrado no estudo HBSC 2018 (15), que apresenta uma prevalência de $11,5 \%$ dos jovens a consumir raramente ou nunca fruta. Resultado semelhante foi obtido no que respeita aos produtos hortícolas, onde a questão relativa ao consumo de produtos hortícolas pelo menos uma vez por dia, apresentou 31,1\% ( $n=101)$ de respostas negativas, superior aos 17,8\% encontrados no HBSC 2018 (15). Ainda que se tenha encontrado uma prevalência de consumo de hortofrutícolas mais elevado do que em estudos anteriores, o consumo pode ser descrito como abaixo do proposto pelas recomendações alimentares para este público-alvo (17).

No que diz respeito ao consumo de bebidas alcoólicas, o presente trabalho apresentou uma prevalência inferior para o consumo raro ou inexistente de bebidas destiladas $(76,3 \%, n=248)$ do que no HBSC $2018(89,4 \%)(15)$, mas superior para o consumo raro ou inexistente de cerveja (83,4\%, $n=271)$. A ingestão de água entre $0,5 \mathrm{~L}$ e $1 \mathrm{~L}$ foi verificada em $37,5 \%(n=122)$ dos participantes, valor semelhante aos 35\% encontrados no HBSC 2018 (15).

As principais bebidas ingeridas ao almoço e ao jantar foram a água (Almoço: 96,9\%, n=279; Jantar: 95\%, $n=264$ ), os refrigerantes (Almoço: 45,5\%, n=131; Jantar: 41,7\%, $n=116$ ) e os néctares de fruta (Almoço: 43,4\%, n=125; Jantar: 38,1\%, n=106). Nos dados do Inquérito Alimentar Nacional e de Atividade Física, IAN-AF 2015-2016 (18), os refrigerantes apresentam o consumo médio mais elevado a seguir à água (88 g/dia) e os adolescentes são o grupo etário com prevalência de consumo diário de um ou mais refrigerantes ou néctares ( $\geq 220 \mathrm{~g} / \mathrm{dia}$ ) mais elevada (42\%, $34 \%$ nas raparigas e $51 \%$ nos rapazes).

Também se encontrou neste estudo uma correlação negativa entre o número de refeições diárias e o percentil IMC/Idade, o que indica que estudantes num percentil IMC/idade mais elevado declaram fazer um menor número de refeições. Esta associação também foi descrita, em outros trabalhos com adolescentes, onde um menor número de refeições diárias associou-se com uma maior prevalência de excesso de peso (19-21).

O consumo de alimentos de elevada densidade energética faz parte do consumo diário habitual dos participantes, ao pequeno-almoço, merendas e ceia. E, 16,3\% refere consumir doces ou guloseimas todos os dias, indo de encontro aos 15,7\% observados no HBSC 2018 (15). Este consumo poderá ser explicado pelo aumento exponencial da disponibilidade destes alimentos nas últimas décadas, e contribui para diversos prejuízos para a saúde, relacionados com a obesidade e respetivas complicações (22).

Neste trabalho encontrou-se um baixo nível de adesão em 9,0\% ( $n=29)$ dos participantes, valor mais elevado que o encontrado por AdelantadoRenau et al. (23) em adolescentes espanhóis (5,2\%, n=14). Num estudo em adolescentes catalães, foi verificada uma associação positiva entre a adesão ao PAM e a prática de atividade física e o menor tempo em atividades sedentárias (tempo de ecrã) (24). No presente estudo, um maior nível de adesão ao PAM associou-se a um estilo de vida mais saudável, onde os jovens inquiridos tomam o pequeno-almoço, fazem um maior número de refeições diárias, consomem sopa ao almoço e jantar, bebem uma maior quantidade de água por dia, passam menos tempo em atividades sedentárias e são fisicamente mais ativos.

Relativamente ao estado nutricional, encontraram-se 19,7\% dos participantes com sobrecarga ponderal, dos quais 17,2\% apresentavam excesso de peso e 2,5\% obesidade. Estes dados estão de acordo com os obtidos no HBSC 2018 (15) que encontraram 15,8\% dos jovens com excesso de peso e 3,1\% com obesidade.
Os rapazes e raparigas apresentaram diferenças estatisticamente significativas para a prática de atividade física. São os rapazes os que mais praticam desporto fora da escola $\left(X^{2}=20,518 ; p<0,001\right)$ e que dedicam mais tempo semanal a esses desportos $\left(X^{2}=12,960\right.$; $p=0,011)$. Estas diferenças entre sexos para a atividade física parece consistente com diversos estudos, em que as raparigas são menos ativas fisicamente (25). A análise da prática de atividade física e a sua relação com o percentil IMC/Idade não mostrou diferenças significativas. Um aspeto que poderá contribuir para a interpretação destes resultados é a energia utilizada na prática de atividade física ser excessivamente compensada com a ingestão alimentar (5).

Os resultados obtidos relativos à higiene do sono vão ao encontro dos obtidos no estudo do HBSC de 2018 (15), em que a percentagem de participantes que referem dormir "mais de 8 horas" durante 0 fim-de-semana é superior comparativamente com dias de semana. Segundo a Sleep Foundation (24) muitos dos adolescentes têm um ritmo irregular de sono durante a semana, havendo uma diferença marcante entre os dias úteis e os fins de semana.

As limitações deste estudo estão relacionadas com a dimensão da amostra, dado que se registou uma taxa de adesão de 52,4\%, tendo em conta o número total de potenciais participantes fornecido pelos agrupamentos escolares. Apesar de se terem assegurado todos os procedimentos possíveis, verificou-se alguma dificuldade no recrutamento dos participantes, dadas as tarefas habituais que desenvolvem na escola e o número de autorizações por parte dos encarregados de educação para participação no estudo. Apesar de se ter alcançado um número de participantes que permita indicar significado estatístico na análise realizada, a taxa de adesão poderá ter comprometido a caracterização e identificação de subgrupos específicos de participantes, com padrões alimentares que careceriam de estudo e/ou intervenção.

\section{CONCLUSÕES}

Este estudo permitiu concluir que os jovens inquiridos apresentam hábitos alimentares e estilo de vida passíveis de melhoria, nomeadamente o consumo do pequeno-almoço, a composição da refeição do almoço, a quantidade de água ingerida ao longo do dia, o consumo de bebidas alcoólicas, o nível de adesão ao PAM, bem como a prática de atividade física. Desta forma, evidencia-se a importância da implementação de estratégias de educação alimentar, que envolvam toda a comunidade educativa, nomeadamente os encarregados de educação e os professores, bem como as entidades de saúde da região, tendo por base o estilo de vida mediterrânico.

\section{AGRADECIMENTOS}

Ao Programa de Cooperação INTERREG V - A Espanha-Portugal $2014-2020$.

À Direção, diretores de turma e jovens participantes das Escolas Secundárias que colaboraram neste estudo.

\section{REFERÊNCIAS BIBLIOGRÁFICAS}

1. Krebs NF, Himes JH, Jacobson D, Nicklas TA, Guilday P, Styne D. Assessment of child and adolescent overweight and obesity. Pediatrics. 2007 Dec;120 Suppl 4:S193228. Disponivel em: DOI:10.1542/peds.2007-2329D.

2. World Health Organization. Nutrition in adolescence: issues and challenges for the health sector: issues in adolescent health and development. World Health Organization, 2005. Disponível em: http://www.who.int/iris/handle/10665/43342.

3. Lourenço A, Nogueira $\mathrm{H}$. Determinantes comportamentais e obesidade entre adolescentes: tendências e variações em território Europeu. Cadernos de Geografia n 33 -2014. Coimbra, FLUC - pp. 141-151. Disponível em: http://dx.doi.org/10.14195/08711623_33_13. 
4. World Health Organization. Guideline: implementing effective actions for improving adolescent nutrition. World Health Organization, 2018 . Disponível em: https://www. who.int/nutrition/publications/guidelines/effective-actions-improving-adolescent/en/. 5. Sobral G. Excesso de peso e estilos de vida na adolescência [Trabalho de dissertação desenvolvido para obtenção do grau de Mestre em Biologia Humana e Ambiente]. Lisboa: Faculdade de Ciências da Universidade de Lisboa. 2013. Disponível em: http:// hdl.handle.net/10451/10331.

6. Dinu M, Pagliai G, Casini A, Sofi F. Mediterranean diet and multiple health outcomes: an umbrella review of meta-analyses of observational studies and randomised trials. Eur J Clin Nutr. 2018 Jan;72(1):30-43. Disponível em: doi:10.1038/ejcn.2017.58.

7. World Health Organization. European Food and Nutrition Action Plan 2015-2020. World Health Organization. 2014. Disponível em: http://www.euro.who.int/en/ publications/abstracts/european-food-and-nutrition-action-plan-20152020-2014.

8. Fleiss, J. L., Levin, B., Paik, M.C. Statistical Methods for Rates and Proportions. Third Edition. John Wiley \& Sons. New York. 2003.

9. Mateus MP. Adesão ao Padrão Alimentar Mediterrânico em jovens no Algarve [Trabalho de dissertação desenvolvido para obter o grau de Doutor em Ciências do Consumo Alimentar e Nutrição]. Porto: Faculdade de Ciências da Nutrição e Alimentação da Universidade do Porto. 2012. Disponível em: https://repositorio-aberto. up.pt/handle/10216/66678.

10. Rito A, Breda J, Carmo I. Guia de avaliação do estado nutricional Infantil e Juvenil. Publicação da Direção Geral da Saúde e do Instituto Nacional de SaúdeDr. Ricardo Jorge. Lisboa. Dezembro 2010. Disponível em: http://www2.insa. pt/sites/INSA/Portugues/Publicacoes/Outros/Documents/AlimentacaoNutricao/ GuiaAvaliacaoEstadoNutricional.pdf.

11. Pickering TG, Hall JE, Appel LJ, et al. Recommendations for blood pressure measurement in humans and experimental animals: Part 1: blood pressure measurement in humans: a statement for professionals from the Subcommittee of Professional and Public Education of the American Heart Association Council on High Blood Pressure Research. Circulation. 2005 Feb 8;111(5):697-716. Disponível em: http://doi:10.1161/01.CIR.0000154900.76284.F6.

12. World Health Organization. BMI-for-age (5-19 years) [página da internet] [acedido a 08 de março de 2019]. Disponível em: https://www.who.int/growthref/who2007_ bmi_for_age/en/.

13. Lurbe E, Agabiti-Rosei E, Cruickshank JK, Dominiczak A, Erdine S, Hirth A, et al. 2016 European Society of Hypertension guidelines for the management of high blood pressure in children and adolescents. J Hypertens. 2016 Oct;34(10):1887-920. Disponível em: doi: 10.1097/HJH.0000000000001039.

14. Gibney MJ, Barr SI, Bellisle F, Drewnowski A, Fagt S, Livingstone B. Breakfast in Human Nutrition: The International Breakfast Research Initiative. Nutrients. 2018 May 1;10(5). pii: E559. Disponível em: doi: 10.3390/nu10050559.

15. Matos MG et al. Relatório do Estudo HBSC 2018: A saúde dos adolescentes portugueses após a recessão - Dados nacionais do estudo HBSC 2018. Lisboa: Aventura Social; 2018.

16. Santaliestra-Pasías AM, Bel-Serrat S, Moreno LA, Bueno G. Consumption of dairy products in youth, does it protect from cardio-metabolic risk? Nutr Hosp. 2016 Jul 12;33(Suppl 4):342. Disponível em: doi: 10.20960/nh.342.

17. World Health Organization. Diet, nutrition and the prevention of chronic diseases: report of a Joint WHO/FAO Expert Consultation. WHO Technical Report Series, No. 916. Geneva: World Health Organization; 2003.

18. Lopes C, Torres D, Oliveira A, Severo M, Alarcão V, Guiomar S. et al. Inquérito Alimentar Nacional e de Atividade Física, IAN-AF 2015-2016: Relatório de resultados. Universidade do Porto, 2017. ISBN: 978-989-746-181-1. Disponível em: www.ian-af.up.pt.

19. Silva FA, Candiá SM, Pequeno MS, Sartorelli DS, Mendes LL, Oliveira RM. et al. Daily meal frequency and associated variables in children and adolescents. J Pediatr (Rio J). 2017 Jan - Feb;93(1):79-86. Disponível em: doi:10.1016/j.jped.2016.04.008. 20. Bibiloni M, Martinez E, Llull R, Juarez MD, Pons A, Tur JÁ. Prevalence and risk factors for obesity in Balearic Islands adolescents. British Journal of Nutrition. 2010; 103: 99-106. Disponível em: doi:10.1017/S000711450999136X.

21. Vik FN, Øverby NC, Lien N, Bere E. Number of meals eaten in relation to weight status among Norwegian adolescents. Scandinavian Journal of Public Health, 2010; 38(Suppl 5): 13-18. Disponível em: doi:10.1177/1403494810378920.

22. Costa CS, Del-Ponte B, Assunção MCF, Santos IS. Consumption of ultra-processed foods and body fat during childhood and adolescence: a systematic review. Public Health Nutr. 2018 Jan;21(1):148-159. Disponível em: doi:10.1017/S1368980017001331. 23. Adelantado-Renau M, Beltran-Valls MR, Esteban-Cornejo I, Martínez-Vizcaíno V, Santaliestra-Pasías AM, Moliner-Urdiales D. The influence of adherence to the Mediterranean diet on academic performance is mediated by sleep quality in adolescents. Acta Paediatr. 2019 Feb;108(2):339-346. Disponível em: doi:10.1111/apa.14472.

24. Fauquet, J., Sofi, F., López-Guimerà, G., Leiva, D., Shalà, A., Sánchez-Carracedo, D., et al. Mediterranean diet adherence among Catalonian adolescents: Socioeconomic and lifestyle factors. Nutricion Hospitalaria, 2016; 33, 1283-1290. Disponível em: doi:10.20960/nh.772.

25. Pérez-Rodrigo C, Gil Á, González-Gross M, Ortega RM, Serra-Majem L, VarelaMoreiras G, Aranceta-Bartrina J. Clustering of Dietary Patterns, Lifestyles, and Overweight among Spanish Children and Adolescents in the ANIBES Study. Nutrients. 2015; Dec 28;8(1). pii: E11. Disponível em: doi: 10.3390/nu8010011.

26. National Sleep Foundation. Teens and Sleep [página da internet] [acedido a 8 de abril de 2019]. Disponível em: https://www.sleepfoundation.org/articles/teens-and-sleep. 